\title{
HISTORIA
}

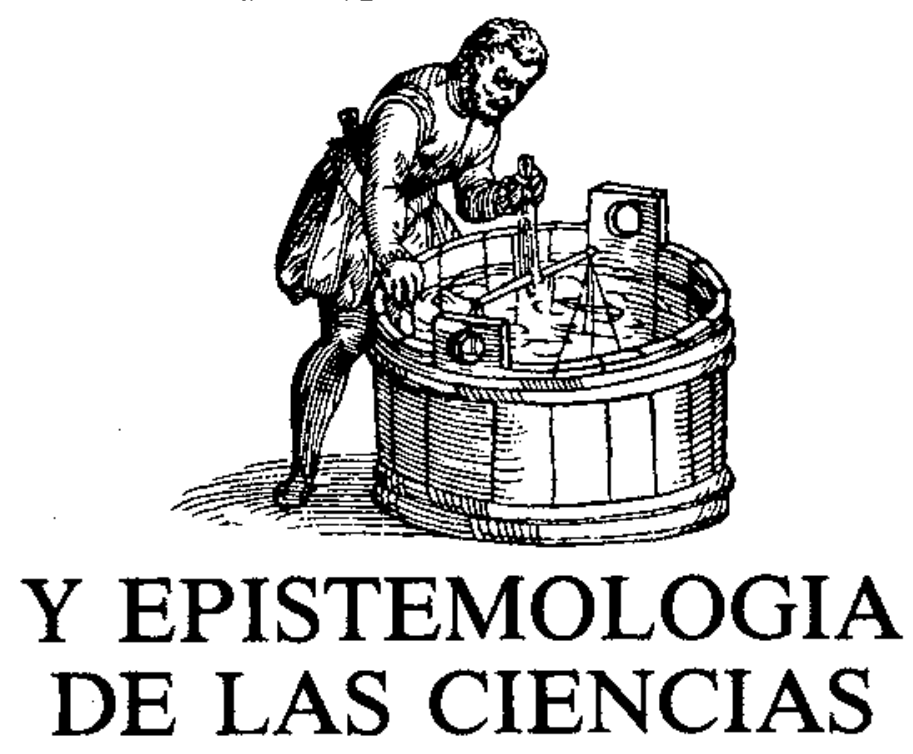

\section{ORIGEN HISTÓRICO DEL TÉRMINO ION}

\author{
GALACHE LóPEz, Ma I., CAMACHO DOMÍNGUEZ, E. y RODRÍGUEz GaRCía, A. \\ Departamento de Química Inorgánica. Facultad de Ciencias. Universidad de Málaga. Campus de Teatinos. \\ 29071 Málaga.
}

Investigación financida por la DGICYT, Proyecto PS87-0075.

\section{SUMMARY}

This paper introduces readers to the origin of the word «ion» through the correspondence between Michael Faraday and William Whewell in the spring of 1834 . Special emphasis is placed on the deep involvement of the two scientists in their discussion and their thoroughness in choosing the most appropriate term, which they knew would be of great historical and educational transcendence.

Al estudiar las ideas de los alumnos acerca del concepto de ion (Camacho 1988, 1989 y 1990), hemos considerado, que la visión histórica podría ayudar a entender las dificultades que se encuentran al pretender introducir este concepto (Saltiel 1985).

La necesidad de hallar una teoría que permita comprender y describir las ideas de los alumnos (Ogborn 1985), nos ha llevado a indagar cómo surgió históricamente este término introducido por Michael Faraday (1791 . 1867) en sus trabajos sobre electrolisis, iniciados por Humphrey Davy (1773-1828).

El estudio de la historia permite conocer en profundidad los marcos teóricos que se han utilizado para interpolar los fenómenos (Izquierdo 1988) y puede servir como 
herramienta para el aprendizaje de algunos conceptos (Caamaño 1987, Gagliardi 1988).

Fl sentido histórico puede presentar una riqueza adicional, ofreciendo como escribe Bent (1977):

«Nada para muchos estudiantes, algo para algunos profesores y mucho para muchos investigadores».

Con estos motivos nos hemos acercado a la persona de Michael Faraday, su obra y su correspondencia.

Faraday es realmente un modelo de científico del siglo XIX. Sc puede decir que fue un autodidacta. Comenzó a trabajar como aprendiz de encuadernación y leía todos los libros que caían en sus manos. Le impresionó, de modo especial, un libro de texto de Jane Marcet Conversaciones en Química. Empezó a experimentar en su casa y asistió a las clases impartidas por Davy. Transcribió una copia de estas lecciones y se la envió al propio Davy, pidiéndole un empleo, que le fue concedido como ayudante de laboratorio en la Royal Institution en 1813. Su talento hizo que se promocionase rápidamente y en 1820 empezó a publicar. Faraday dió a Crooke este consejo: «Trabaja, termina, publica», que debió ser también su propio lema.

A partir de aquí comienza su trabajo científico, duro, perseverante, sistemático, sobre numerosas y variadas cuestiones de la Física y de la Química. Fundamentales son sus aportaciones al descubrimiento del electromagnetismo y los estudios sobre electrolisis.

Faraday recogió la mayoria de sus publicaciones en: «Philosophical Transactions» $y$ «Philosophical Magazine", en dos libros (A y B); un libro de separatas (C), algunas de sus conferencias publicadas por otros autores (D y E), y en sus notas y diario de laboratorio (F). Todo ello se relaciona a continuación (Partington 1969):

A. Experimental Researches in Electricity, 3 vols. (1839, 1844 y 1855$)$.

B. Experimental Researches In Chemistry and Physics, (1859).

C. Chemical Manipulation, $(1827,1829,1830$ y 1842).

D. The Chemical history of Candle. Editado por William Crookes (1861).

E. The Subject Matter of a Course of Six Lectures on the Nonmetalic Elements. Editado por J. Scoffern (1853).

F. Faraday's Diary being the Various Philosophical Notes of Experimental lnvestigations made by Michael Faraday during the years $1820-1862 \ldots$ under the editorial supervision of Thomas Martin. 7 vols. and index.

Son muy interesantes las «scries", como él mismo las llamó, de su libro Experimental Researches in Chemistry and Physics (B). La serie VII recoge sus aportaciones sobre la descomposición electroquímica y es especialmente interesante desde nuestra perspectiva.

Faraday introdujo toda una terminología que se sigue utilizando en la actualidad: electrolisis, electrolitos, electrodos, ánodo, cátodo, anión, catión, ion... Algunos de estos términos le fueron sugeridos por Willian Whewell (1794-1866) a petición del mismo Faraday.

Afirmó que la corriente eléctrica es transportada a través del material fundido o de la disolución por uentidades» que denominó «iones». Los que viajaban al ánodo eran aniones, y los que iban al cátodo, cationes. Llegó a identificar los iones como partículas que transportaban la electricidad, pero, sin abordar su naturaleza.

Por primera vez en la Historia de la Ciencia aparecen estos términos, que han jugado un papel decisivo en el conocimiento de numerosos fenómenos de la Física, Química y Biología. Resulta curioso constatar que estos conceptos, cuyo significado nos resulta hoy tan claro y familiar, fueron en su momento objeto de numerosas precisiones, revisiones, cambios, idas y venidas por parte de los científicos que les dieron vida, hasta llegar al significado que hoy tienen.

Produce un gran asombro y admiración asomarse a la correspondencia que Faraday mantiene sobre el tema en 1834 , con otros colegas, especialmente con W. Whewell, profesor del Trinity College de Cambridge. Matizan el sentido de cada palabra y de lo que con ella quieren dar a entender Es como adentrarse en el trabajo serio y minucioso de estos hombres y ver lo importante que para ellos es el intercambio de opiniones, en suma, la comunicación entre aquéllos que se dedican a la investigación científica, incluso antes de la publicación de sus trabajos.

Transcribimos a continuación algunos párrafos de esta correspondencia, dado su interés para esclarecer el origen del término ion que nos ocupa (Pearce 1971). La primera de estas cartas la escribi6 Faraday a Whewell el 24 de abril de 1834 .

Busco algunos nombres para expresar mis experiencias en electricidad. Así, un cuerpo descompuesto por la corriente eléctrica yo lo llamo "electrolito" y si es descompuesto electroquímicamente digo que está uelectrolizado". Los llamados "polos" de la batería, los llamo "electrodos", y no son sólo superficies de metal sino a veces, agua y aire, a los cuales se puede aplicar el término "polo» sin que resulte para éste un sentido nuevo.

Los electrolitos deben constar de dos partes, que, durante la electrolización, una va en un sentido y la otra en el

Figura 1

Representación del proceso de electrolisis dada por M. Faraday a W. Whewell en su carta del 24 de abril de 1834 (Pearce 1971).
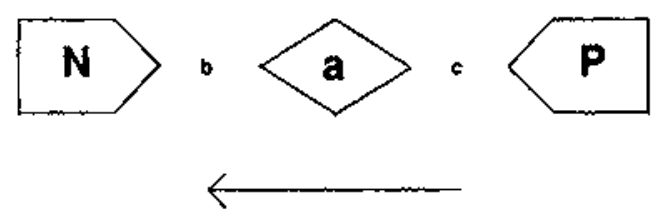
contrario, hacia los "electrodos" o "polos» donde son liberados. Yollamo "zetodos" a los constituyentes de los "electrolitos".

Estoy satisfecho con algunos de estos términos, pero no con otros que he utilizado Es esencial para mi poder referirme a las dos superficies del cuerpo descompuesto, por las que entra y sale corriente, sin referirme al mismo tiempo a los «electrodos».

Asi, a es el cuerpo que se descompone, $N$ y $P$ los polos negativo y positivo, que pueden estar o no en contacto con a en los puntos b y c. (Fig. 1).

Admitiendo el modo usual de expresión y hablando de una corriente eléctrica, procedente del polo positivo, $P$, hacia el polo negativo, $N$, a través de a, un amigo sugirió -y yo lo he utilizado- el término "eisodo" para cy "exodo" parab. Serian los puntos por donde los «zetodos" se unen a los «polos». Quisiera dos nombres para $c$ y $b$, a los que pueda referirme sin involucrar ninguna teoria acerca de la naturaleza eléctrica.

Buscando una referencia, he encontrado que el magnetismo terrestre se debe a corrientes eléctricas cuyas lineas irían, según nuestro modo de expresión, de Este a Oeste. Haciendo un paralelismo, en una porción de agua descompuesta por la corriente eléctrica, se podríaconsiderar una corriente eléctrica como circulando alrededor de la tierra; el oxigeno iría hacia el Este y el hidrógeno hacia el Oeste. Pienso que entonces podría llamar "eastodo" $a$ a y «westodo" $a b$. O bien, «oriodo" y «occiodo». Pero no son nombres que un escolar pueda recordar fácilmente.

Usted puede ayudarme a buscar dos buenos nombres, que no dependan sólo de la dirección de la corriente, y a los que pueda añadir los prefijos "zeto" o «zet", para expresar la clase de "zetodo" al que pertenecen.

Llama la atención que Faraday se preocupe porque «los escolares» puedan recordar estos términos fácilmente. Aparece en su carta una inquietud de carácter didáctico: que el término sea de fácil lectura, claro, preciso, etc.

En carta fechada el 25 de abril de 1834 , Whewell contesta a Faraday y le sugiere por primera vez los nombres «ánodo» y "cátodo».

He considerado los dos términos que usted quiere que sustituyan a "eisiodo" y "exodo", y estoy dispuesto a recomendarle los de "anodo" y "catodo». Estas pala* bras pueden sinificar "camino hacia el Este» $y$ «camino hacia el Oeste», que indican de forma más sencilla lo que usted pretende. Son dos palabras griegas genuinas y no acuñadas a propósito. Si no está satisfecho con ellas, puedo ofrecerle uno o dos pares más de nombres. Por ejemplo: "dexiodo"y "sceodo" ( sskaiodo" si pre. fiere) pueden utilizarse para indicar Este y Oeste, aunque su significado original sería «derecha» e «izquierda" Otro par de nombres: "orthodo" y "anthodo" que significan "camino directo y opuesto"...

Pienso que "catodo" y "anodo" son desde luego mejores [...] Ya le he dicho que me gustan la mayoría de sus palabras nuevas, pero hay una que excluyo de mis elogios y es «zetodo». Éstas son mis objeciones: esta palabra agrupada con las otras de la misma terminación, puede esperarse que indique una modificación de "electrodo", como "eisiodo"y "exodo", o "anodo" y "catodo" Pero en lugar de esto "zetodo" es una noción diferente, por lo que necesita usted una palabra nueva que pueda recordar que la descomposición de la que usted habla es una descomposición electrolítica.

Quizás la palabra griega "stecheon" (o "stoicheion" respondería a este propósito y además tiene ya un lugar en nuestrolenguaje cientifico: "stoicheiometry". Mientras la palabra "zetodo" terminada en "odo" sinifica "camino", "stecheon" se deriva de una palabra que sinifica "ir en fila y es más apropiada, ya que los elementos «zetodos» son dos cosas que van o intentan ir por caminos opuestos $Y$ debo añadir que la referencia debe hacerse al proceso de descomposición por el cual se obtienen estos elementos. Usted puede llamar a su "zetodo" un «electrostecheon" porque ha sido obtenido por procesos electroquímicos, [...] Yoopinoque "anastecheon" y "catastecheon" pueden indicar los dos «stecheones».

Y termina: ... mi carta se hace muy larga y quiero recapitular: "anodo", "catodo», "zetanodo" y "zetaca. todo" cumplen todos los requisitos. "Anodo" "catodo", "anastecheon" $y$ "catastecheon" son los que yo prefiero.

En la contestación de Faraday a Whewell el 3 de mayo de 1834 , le agradece los nuevos nombres sugeridos, y dice: "Dexiodo" $y$ "Skeiodo», con su significado Este y Oeste son buenos, me parece que suena mejor "anodo" $y$ "catodo", pero a quien se lo he comentado piensa que el significado de "anodo" puede ser "no camino" (partícula a). "Stechion" parece duro de sonido [...] Es posible que en este tiempo se me ocurra una palabra más corta que "stechion".

Evidentemente Faraday se ha dejado influir por la brillante aportación de Whewell, pero lejos de dar por terminado este asunto, ha insistido en algunas palabras en las que Whewell no está muy de acuerdo e invita a reducir el tamaño de stechion (no de stecheon, ni de stoicheion).

Whewell reponde a Faraday inmediatamente, el 5 de mayo de 1834, con una carta que se transcribe a continuación casi completa por su interés y aportación al debate sobre estos términos. Es bastante definitiva en la decisión que toma, finalmente, Faraday.

Estoy de acuerdo con usted en que "stechion" o "stecheon" son unas palabras duras por su lectura y por las letras que las componen, y deseo mucho que encuentre una mejor para su propósito. Creo que puedo sugerirle una más, pero antes quisiera que recordara la sugerencia de "anodo" y "catodo» que le ofrecí anteriormente. Es obvio que estas palabras son mucho más simples que las que usted está probando y esta ventaja será muy impor. tante cuando las palabras queden establecidas, cosa que no dudo ocurrirá con su publicación.

Ante la objeción sobre anodo, no creo que sea de suficiente peso, para hacerle dudar. "Anodo» y «cato- 
do" realmente sinifican en griego "un camino hacia arriba» y "un camino hacia abajo»; y el significado de "anodo", de acuerdo con su analogía en el lenguaje griego, no puede ser "no camino». Es cierto que el prefijo "an" delante de adjetivos que comienzan con vocal da un sentido negativo, pero no ante sustantivos [...] "Anodo» no puede pensarse que signifique "no camino».

Insiste con más argumentos sobre la objeción y continúa: Si Ud. toma "anodo» y "catodo», yo le propondria para los dos elementos resultantes de la electrolisis los términos de "anion" y "cation", que son participios neutros que significan uel que va hacia arriba y el que va hacia abajo y para los dos juntos el término "ion», en vez de «zetodo»o «stechion».

Esta palabra no es sustantivo en griego, pero se puede tomar asi y estoy convencido de que la brevedad y simplicidad de los términos hará que en umenos de quince días» su aceptación sea universal. «Anion» es el que va al "anodo" y "cation" el que va al "catodo».

Su párrafo podría quedar así: "Nos proponemos llamar al que va hacia el Este «anodo»*, y al que va al Oeste "catodo"** (...) Propongo distinguir las partes que proceden de la descomposición del cuerpo llamando "anion»*** a la que va al "anodo» y "cation»**** a la que va al "catodo". Y cuando tenga ocasion de hablar de las dos juntas las llamaré «iones».

* $\quad \alpha \vee \alpha$ : hacia arriba, o $\delta$ o :camino.

** $\quad x \alpha t \alpha$ : hacia abajo, o $\delta 0$ : camino.

*** avi o v: lo que sube.

$* * * *$ K $\alpha \tau 10 v$ : lo que baja.

Faraday escribe a Whewell el mismo 5 de mayo de 1834 : Con la esperanza de que llegue esta carta antes de que usted me escriba, quiero mencionarle dos nombres que pueden sustituir a «eisodo» $y$ «exodo», serian «voltodo» y "galvanodo».

Mi amigo el Dr. Nichols me propone "alphodo» y «bethodo". Las partes serian "voltastechion", "galvastechion" o "alphastechion" $y$ «betastechion".

Whewell insiste de nuevo en su carta del 6 de mayo: Estoy más y más convencido que "anodo" y "catodo" son los mejores términos para establecer una comparación entre los clectrodos, cosa que no ocurre con "alphodo» $y$ ubetodo", que menciona; y menos aún con "voltodo" y "galvanodo", que son ostensiblemente arbitrarios, con dos desventajas: es muy dificil recordar cuál es cual en el cuerpo que se descompone y no se puede estar seguro que posteriores investigaciones no aporten alguna incongruencia histórica con esta referencia a Volta y Galvani.

Estoy convencido de que las palabras correctas son "anodo" y "catodo", pero no quisiera que por esta propuesta tuviera alguna diferencia u oposición con el Dr. Nichols.

Finalmente Faraday acepta esta nomenclatura y escribe a Whewell el 15 de mayo de 1834 en una carta muy sincera y humana, que, entre otras cosas, dice: $₫ D e b o$ agradecerle su gran bondad en este asunto de los nombres que le he consultado. Esperaba encontrarle el sábado en Kensington para expresarle mi reconoci. miento. He aceptado sus consejos y los nombres que he utilizado son: "anodo", "catodo", "cationes", "uniones»e «iones». Es verdad, que yo tenía objeciones hacia ellos y me encontraba como aquel hombre que iba con su hijo y el asno, que quería complacer a todo el mundo. Pero, cuando to he pensado de nuevo, amparado en su autoridad, ha vido luminoso observar cómo toda objeción se ha desirthecids.

Estoy plenamente sutisfecho con la facilidad de expresion que me proporcionan las nuevas palabras y le seré deudor por la ayuda que me ha prestado..

Éste fue el final o más bien el principio de esta terminología. Es evicuente que Faraday no sólo pretendía expre sar de forma sencilla y precisa sus ideas, también tenía una intencionalidad didáctica al elegir estas palabras.

Resulta también interesante ver cómo en el diario de Faraday se van justificando y modificando, a la vez, los nombres. Piensa y escribe el significado asignado a cada uno de ellos, algunos anteriores a la correspondencia que sostiene con Whewell y otros a raíz de ellat.

«Equivalente electroquímico» aparece el 23 de septiembre de 1833. El 19 de diciembre del mismo año, Furaday utiliza la palabra «electrobeids» para «iones». «Electrodom, el 17 de diciembre de 1833 , frecuentemente seguido de positivo y negativo. Escribe en el diario: En lugar del término «polo» propongo usar «electrodo».

Para Faraday el «anodo» era en principio el extremo negativo del «electrolito» cn contacto con la superficie positiva del metal, por la que la corriente eléctrica entra en el «electrolito». Algo semejante ocurría en el «catodo».

En febrero de 1834 utiliza la palabra «zetodo». Los nombres de Whewell aparecen por primera vez el 13 de mayo de 1834 .

Faraday realizó un estudio cuidadoso de la electrolisis del agua acidulada con ácido sulfúrico en aparatos con electrodos de platino. Utilizó cinco modelos de aparatos. (Partington 1969).

Los gases unas veces se recogían separados y otras mezclados. Sin usar galvanómetros ni ningún otro tipo de instrumento de medida de corriente, probó que la cantidad de agua descompuesta es independiente del tamaño de los electrodos, forma de los tubos, de lat intensidad de corriente (por la que unas veces entendía voltaje y otras densidad de corriente), y de la fuerza del ácido.

Como resultado de estos experimentos, estableció las leyes de la electrolisis, aportando numerosos datos cuantitativos.

Utiliza por primera vez el nombre de «voltámetro» para sus aparatos en septiembre de 1833 , en su edición de «Experimental Researches». 
Estudió también las reacciones secundarias en la electrolisis con resultados muy interesantes y en muchos casos correctos.

Después de resumir su nomenclatura (en la que por un desliz coloca el hidrógeno con los aniones cloro, oxígeno, ctc.), Faraday resume sus resultados en once proposiciones, no sin antes escribir: Espero no incluir en ellos ningún error serio.

\section{Se pueden sintetizar así estas proposiciones:}

I. Un ion solo, sin estar en combinación con otro, no tendría tendencia a pasar a los electrodos, sería independiente al paso de la corriente a menos que esté compuesto de iones más elementales, y sea sujeto de descomposición.

II. Si un ion está combinado con otro totalmente opuesto en su reactividad química, es decir, si un anión se combina con un catión, ambos viajarán el uno al ánodo y el otro al cátodo por la descomposición del cuerpo.

III. Si un ion va hacia uno de los electrodos, el otro debe ir simultáneamente al otro electrodo, aunque por t:na acción secundaria, no lo parezca.

IV. Un cuerpo que se puede descomponer directamente por la corriente eléctrica, es decir, un electrolito, debe constar de dos iones y debe proporcionarlos durante la acción de descomposición.

V.Para un electrolito, compuesto de dos iones elementales, parece ser un hecho que sólo un equivalente químico de los iones clementales puede ir hacia los electrodos, y no múltiplos.

Vi. Un cuerpo que no se puede descomponer en sas elementos, al realizar la electrolisis, actúa como si sus iones fueran al ánodo o al cátodo, pero no libera sus elementos, excepto cuando hay reacción secundaria.

VII. La naturaleza de la sustancia que forma el electrodo no causa diferencia en la electrodescomposición...

VIII. Una sustancia que, al ser utilizada como electrodis, puede combinarse de nuevo con el ion liberado, creo qlie es también un ion, y se combina en cada caso con ła cantidad representada por su equivalente electroquímico.

IX. Los iones compuestos noestán necesariamente formades de equivalentes electroquímicos de iones simples...

$X$. Los equivalentes electroquímicos son siempre invariables. Así, el mismo número que representa el equivalente de una sustancia A cuando se separa a partir de una sustancia B, también representaráa a A cuando proceda de una tercera sustancia $\mathrm{C}$.

XI. Los equivalentes electroquímicos coinciden y son los mismos que los equivalentes químicos ordinarios.

De estas proposiciones la $\mathrm{V}$ es incorrecta, la VI es oscura, y el resto puede afirmarse que son correctas considerando la terminología del momento. Hay que decir que lo que Faraday llama iones no son los iones de hoy: sus iones son sales, óxidos metálicos, amoniaco, anhídridos de ácido, tanto como metales, oxígeno y cloro.

El hecho importante es que Faraday tuvo el mérito de establecer las primeras relaciones cuantitativas en electroquímica y se aventuró a hablar de iones. Sin embargo, durante el medio siglo siguiente, nadie trabajó seriamente sobre la naturaleza de estos iones; aunque sí hubo aportaciones muy valiosas (Asimov 1988, Mason 1986).

Así Grothus admitió la existencia, en toda disolución, de átomos o radicales cargados. Esta hipótesis iónica, reafirmada posteriormente por Clausius y Helmholtz, y finalmente por Arrhenius, tuvo en un principio gran resistencia y oposición. Pero no sólo ha conducido a la explicación de la electrolisis y al transporte de la electricidad en los conductores de segunda clase, es decir, en las disoluciones (Hittorf 1853, Kohlrausch 1879), sino que ha hecho posible la aplicación de la ley de acción de masas a los electrolitos y la producción de energía eléctrica en las pilas galvánicas (Nernst 1889). Además ha proporcionado la certidumbre de la estructura eléctrica de la materia y con ello ha contribuido eficazmente a la creación de la teoría de los iones y a la génesis de la ideas actuales acerca de la constitución del átomo y de las moléculas (Felix Cid et al. 1982).

La figura clave de esta etapa posterior es otro gran maestro: Svante August Arrhenius (1857-1927) que, con la tcoría de la disociación electrolítica, presentada en su tesis doctoral, dio un sal to importante desde Faraday a nuestros días. Sin olvidar la aportación que el estudio del estado sólido ha realizado en este sentido, al demostrar la existencia previa de los iones. Adentrarse en la personalidad científica de Arrhenius sería objeto de otro trabajo, que merece la pena emprender.

Como conclusión final, cabe destacar el interés y esfuerzo realizado por Faraday para que su terminología fuese etimológicamente adecuada, precisa en cuanto a su significado y fácil de recordar. Es, sin duda, importante resaltar su buen quehacer científico, incluso en detalles como la clección de las palabras que va a utilizar para expresar sus ideas sobre la Ciencia que estáa construyendo.

\section{REFERFNCIAS BIBLIOGRÁFICAS}

ASIMOV, I., 1988. Breve historia de la Quimica. (Alianze: Editorial: Madrid).
BENT, H.A., 1977. Uses of history Chemistry, Journal of Chemical Education, 54(8), p. 463. 
CAAMAÑO, A., MAESTRE, C., MAYOS, C. y VENTURA T., 1987. La Historia de la Química: una herramienta útil para el aprendizaje de los conceptos qufmicos, Enseñanza de las Ciencias, $\mathrm{n}$. extra, pp. 161-162.

CAMACHO, E., BLANCO, A., GALACHE, I., PRIETO, T. y RODRÍGUEZ, A, 1988. Construcción de un mapa concepiual referido al término ion. Bienal de la Real Sociedad Española de Química. Murcia.

CAMACHO, E., GALACHE, I. y RODRÍGUEZ, A., 1989. Los mapas conceptuales y el aula, Enseñanza de las Ciencias, $\mathrm{n}$. extra, pp. 151-152.

CAMACHO, E., GALACHE, I. y RODRÍGUEZ, A., 1990, «Las ideas de los alumnos y los libros de texto. Una aproximacion al concepto de ions. Cambio educativo y desarrollo profesional Actas de las VII Jornadas de Investigación en la Escuela. (Servicio de Publicaciones de la Universidad de Sevilla: Sevilla).

CID, F. et al., 1982. Historia de la Ciencia. (Planeta: Barcelona).

GAGLIARDI, R., 1988. Cómo utilizar la historia de las Cien- cias en la enseñanza de las Ciencias, Enseñanza de las Ciencias, 6(3), pp. 291-296.

IZQUIERDO, M., 1988. La contribuctón de la Historia de la Química a la Didáctica de la Química. XXII Bienal de la real Sociedad Española de Quimica. Murcia.

MASON, S, F. 1986. Historia de las Ciencias. Vol.4, La Ciencia del siglo XIX. (Alianza Editorial: Madrid).

OGBORN, J, 1985. Understanding student's understandings: An example from dinamics, European Sournal of Science Education, 7, p. 141.

PEARCE, L. (ed.), 1971. The selected correpondence of Michael Faraday. (Ed. by L. Pearce Willians. The Royal Instifution of Great Britain. University Press: Cambridge).

PARTINGTON, J.R., 1969. A History of Chemistry, Vol. 4. (MacMillan St. Martin Press: London).

SALTIEL, E. y VIENNOT, L., 1985. ¿Qué aprendemos de las semejanzas entre las ideas históricas y el razonamiento espontáneo de los estudiantes?, Enseñanza de las Ciencias, 3(2), pp. 137-144. 